\title{
Electromagnetic navigation bronchoscopy as an adjunct diagnostic tool in the Danish lung cancer diagnostic pathway: an initial retrospective single centre series
}

\author{
Michael Stenger ${ }^{1}$, Sally Zoorob ${ }^{2}$, Abdinasir Ahmed Hussein ${ }^{2}$, Jens Eckardt $^{1}$ \\ ${ }^{1}$ Department of Cardiothoracic Surgery, Odense University Hospital, Odense, Denmark; ${ }^{2}$ Faculty of Health Sciences, University of Southern \\ Denmark, Odense, Denmark \\ Contributions: (I) Conception and design: All authors; (II) Administrative support: M Stenger, J Eckardt; (III) Provision of study materials or patients: \\ M Stenger, J Eckardt; (IV) Collection and assembly of data: All authors; (V) Data analysis and interpretation: All authors; (VI) Manuscript writing: \\ All authors; (VII) Final approval of manuscript: All authors. \\ Correspondence to: Michael Stenger. Department of Cardiothoracic Surgery, Odense University Hospital, J.B. Winsløws Vej 4, 5000 Odense C, \\ Denmark. Email: michael.stenger@rsyd.dk.
}

Background: The performance of electromagnetic navigation bronchoscopy (ENB) is reported with substantial variation, which may question its clinical usefulness. However, ENB may hold its true value when used as an additional minimal invasive diagnostic option before potential surgery in selected diagnostically challenging patients where traditional diagnostic methods have failed. We evaluated the safety and performance of ENB when used as an adjunct diagnostic tool in the Danish lung cancer diagnostic pathway (DLCDP) and its ability to reduce surgical diagnostic procedures.

Methods: A retrospective study was performed on eighty-two consecutive patients at Odense University Hospital from June 2016 to March 2018 with diagnostically challenging pulmonary lesions referred for ENB as an adjunct diagnostic procedure under the DLCDP. Patients with benign or inconclusive ENB pathology were either referred for further biopsies, surgery or repeated computer tomography (CT) scans for surveillance purposes.

Results: Eighty-one ENB procedures were performed in 80 patients. In $87.7 \%$ of the cases previous diagnostic methods had been unsuccessful. The mean target diameter was $1.55 \mathrm{~cm}$ and the average followup duration was 11 months. The diagnostic accuracy was $75 \%$, while the diagnostic yield/sensitivity, negative predictive value and negative likelihood ratio was $51 \%, 67 \%$ and 0.49 , respectively. No pneumothoraces and only one intrapulmonary haemorrhage was recorded, which was managed conservatively. The learning curve revealed an increase in diagnostic accuracy from $67.5 \%$ to $82.9 \%$ when comparing the first 40 ENB procedures with the last 41 procedures, however, this was not statistically significant (p-value: 0.11).

Conclusions: ENB was found to be a safe procedure with an acceptable diagnostic accuracy and yield in highly selected diagnostically challenging patients. The introduction of ENB carried a notable learning curve but proved to be a valuable adjunct diagnostic option in the DLCDP, which may help to reduce the number of potentially unnecessary or harmful surgical procedures in frail patients.

Keywords: Electromagnetic navigation bronchoscopy (ENB); diagnostic accuracy and yield

Submitted Mar 09, 2020. Accepted for publication Aug 05, 2020.

doi: $10.21037 /$ jtd-20-1236

View this article at: http://dx.doi.org/10.21037/jtd-20-1236 


\section{Introduction}

Incidental findings of indeterminate pulmonary lesions are on the rise due to the growing use of computed tomography (CT) scans, extensive cancer follow-up protocols and emerging lung cancer screening programs. In Denmark, since 2008 all findings of indeterminate pulmonary lesions have been fast-tracked through a government initiated uniform diagnostic pathway, named the Danish lung cancer diagnostic pathway (DLCDP) (1). The majority of patients with suspicious lesions are diagnosed successfully using traditional minimally invasive diagnostic methods, such as standard flexible bronchoscopy, endobronchial ultrasonography (EBUS), peripheral endobronchial ultrasonography (pEBUS) and CT- or ultrasound (US)guided transthoracic needle aspiration (TTNA). Timely and optimal management, however, remains a challenge in patients where the traditional minimal invasive diagnostic methods are unsuccessful or not amenable. The diagnostically challenging patients typically present with small or ill-defined lesions located in the central zones of the lung beyond the reach of the bronchoscope or with close anatomical proximity to the heart, major vessels, diaphragm or a previously resected area of the lung. Conventionally, these cases are referred to a cardiothoracic unit for a diagnostic thoracoscopic resection, but some patients may also have substantial comorbidities, including poor lung function and performance status, history of previous cardiothoracic surgery or radiotherapy etc. Factors which significantly increase the risk of complications and even fatal outcomes of potentially futile surgical procedures $(2,3)$.

Fortunately, new bronchoscopic diagnostic technologies like electromagnetic navigation bronchoscopy (ENB) has become more widespread over the past decade, which may help to reduce the need for surgical diagnostic procedures in frail patients $(4,5)$. ENB has the capacity to reach the central and peripheral zones of the lung, however, the diagnostic yield of ENB has been reported with considerable variation mainly due to heterogenic patient selection and differences in hospital setups (+/fluoroscopy, +/- rapid on-site examination (ROSE), general anaesthesia versus sedation etc.) (6-9). Additionally, ENB is associated with a significantly higher cost per procedure than traditional diagnostic methods (10). Therefore, we anticipated that the most fitting and optimal use of ENB, in the Danish setting, was to implement it as an extra minimally invasive diagnostic tool in selected diagnostically challenging patients where traditional minimal invasive diagnostic methods have failed.

The objective of this study was to evaluate the diagnostic yield, accuracy and safety of ENB when added to the DLCDP as an adjunct diagnostic option if other traditional diagnostic methods had failed or were unfit for use. Furthermore, we included a learning curve of this initial series and comment on the potential impact of introducing ENB on the need for surgical diagnostic procedures. We present the following article in accordance with the STARD reporting checklist (available at http://dx.doi.org/10.21037/ jtd-20-1236).

\section{Methods}

ENB was introduced at the Department of Cardiothoracic Surgery at Odense University Hospital in June 2016 in order to provide an extra diagnostic option in selected diagnostically challenging cases. This study was performed as a part of a quality assessment project; hence no informed consent of the participants was required. The investigation covered a retrospective investigation of the diagnostic yield, accuracy, safety and the learning curve of the initial consecutive ENB series from June 2016 to March 2018. The study was conducted in accordance with the Declaration of Helsinki (as revised in 2013) and approved by the local Ethics Committee at Odense University Hospital (case number: 18/12554).

\section{Patient selection and data collection}

All included patients were discussed at multidisciplinary team (MDT) meetings comprising a thoracic surgeon, a respiratory physician, a pathologist, an oncologist, a nuclear medicine physician and an interventional radiologist. Indications for ENB were primarily based on the following two criteria (I) previous unsuccessful traditional diagnostic procedures (flexible bronchoscopy, EBUS/pEBUS, US/CTguided biopsy) or (II) consensus that traditional diagnostic procedures were considered low yield or highly likely to fail. Obviously, considerations regarding tumour size and location, patient co-morbidities including emphysematous changes around small peripheral lung lesions, pulmonary function, and the clinical probability of malignancy were all part of the MDT discussion before offering ENB.

Patients with benign or inconclusive ENB pathology were either subjected to additional biopsy attempts with EBUS or pEBUS, surgical resections or followed with repeated CT scans based on a new MDT decision. 
Pulmonary lesions with consistent benign pathology on several consecutive biopsies or with no signs of growth or morphological changes on repeated CT scans were considered as "true negative". In contrast, if a later biopsy or surgical resection showed malignancy or if a CT scan revealed growth or morphological changes consistent with lung malignancy, we considered this a "false negative".

Patient and lesion characteristics, including previous malignancies and previous diagnostic procedures performed, target size, lesion location, and ENB pathology results were recorded.

\section{ENB}

We used the superDimension Navigation Version 7.1 (Medtronic, Plymouth, MN, USA), which consists of four key elements: (I) planning and procedure software; (II) an electromagnetic field; (III) a steerable and locatable guide within an extended working channel (EWC); (IV) various biopsy and marking tools (needle aspiration, brushes, forceps, fiducial markers, dye etc.). The phases and techniques of the ENB procedure have previously been described in depth by Leong and colleagues (11). In brief, we uploaded a formatted CT or PET-CT scan to the iLogic Software, generating a three-dimensional (3D) reconstruction of the lungs and airways. The lesion of interest was marked creating a 3D target. The closest and most suitable bronchus was found and followed backwards to the trachea, creating a delineated virtual pathway. The planned pathway was saved and brought to the operating theatre. All procedures were performed under general anaesthesia using a standard flexible video bronchoscope (Olympus EVIS EXERA II BF-IT180) via a laryngeal mask or an endotracheal tube size 8 or larger. Following standard registration and synchronization, the bronchoscope was advanced as far as possible along the planned pathway and wedged into the sub-segmental ostium leading to the target. The remaining part of the pathway was completed by advancing and steering the EWC with the locatable guide inserted under virtual guidance. Fluoroscopy was not used to confirm the position of the EWC before sampling because we wanted to simplify the setup as much as possible. Likewise, a simple sampling protocol containing three steps was used in the following order: (I) minimum three vacuum needle aspirations, (II) minimum three forceps biopsies, and finally (III) flushing with $20 \mathrm{~mL}$ saline through the EWC at the target site and aspiration into a vacuum syringe. ROSE of the samples was not feasible at our institution.

\section{Statistics}

Descriptive statistics are presented as a mean [standard deviation (SD) or range] or as frequencies (\%). The following definitions were used to calculate (I) diagnostic yield/sensitivity $=[$ true positives $/$ true positives + false negatives)]; (II) negative predictive value $(\mathrm{NPV})=[$ true negatives /(true negatives + false negatives)]; (III) diagnostic accuracy $=[($ true positives + true negatives $) /$ all ENB pathology]; (IV) negative likelihood ratio $=(1-$ sensitivity $) /$ specificity.

Specificity [true negatives/(false positive + true negatives)] was $100 \%$ by definition because "false positives" ENB pathology was considered impossible or 0 .

A chi-squared test was used for comparison of the learning curve between two groups. The relationship between previous cancer diagnosis and malignant ENB pathology was explored through logistic regression analysis. Both analyses were conducted post hoc using Stata/IC version 16, (Statacorp).

\section{Results}

Eighty-two patients were referred for ENB in the period from June 2016 to March 2018. Two patients were excluded leaving 80 patients with the basic characteristics as shown in Table 1. As illustrated in Figure 1, one patient was excluded due to anaesthetic problems and the second patient had previously undergone a lung transplant and was for this reason transferred to another hospital. A total of $81 \mathrm{ENB}$ procedures in 80 patients were performed because ENB was performed twice in one patient.

Fifty-eight of the patients had a history of previous cancer $(71.6 \%)$ with non-small cell lung carcinoma (NSCLC) being the most common $(18 / 58 ; 31 \%)$. Moreover, the majority of the patients $(87.7 \%)$ had prior to the ENB procedure been subjected to different unsuccessful diagnostic biopsy attempts (Table 1).

The characteristics of all pulmonary lesions biopsied are displayed in Table 2 showing that the majority of the lesions were located in the upper lobes $(67.9 \%)$ and all targets were generally small in size with a mean diameter of $1.55 \mathrm{~cm}$ [standard deviation (SD): 0.4 ] with only 8/81 (9.9\%) targets $>2 \mathrm{~cm}$ in diameter.

The ENB pathology result revealed a malignant diagnosis in 21 out of 81 procedures $(25.9 \%)$ with NSCLC being the most common diagnosis $(n=18)$. Nevertheless, the largest proportion of ENB procedures showed either 
Table 1 Patient characteristics $(\mathrm{n}=80)$

\begin{tabular}{lc}
\hline Characteristic & Data \\
\hline Age, mean [range] in years & $69[38-88]$ \\
Female, n (\%) & $58(48.1)$ \\
Previous history of cancer, n (\%) & 18 \\
NSCLC & 9 \\
Skin & 8 \\
Gl & 3 \\
Head/neck & 8 \\
Renal/urinary tract & 8 \\
Breast /gynaecological & 3 \\
Prostate & 1 \\
Lymphoma & $71(87.7)$ \\
Prior diagnostic attempts, n (\%) & 53 \\
Bronchoscopy/EBUS/pEBUS & 16 \\
CT-guided TTNA & 2 \\
US-guided TTNA & 8 \\
\hline
\end{tabular}

Displays basic characteristics of all included patients including types of previous cancers and diagnostic attempts. NSCLC, nonsmall cell lung carcinoma; EBUS, endobronchial ultrasonography; pEBUS, peripheral endobronchial ultrasonography; TTNA, transthoracic needle aspiration. benign (fibrosis, inflammation/pneumonia or atypical cells) or inconclusive pathology $(60 / 81 ; 74.1 \%)$. Based on a new MDT decision, approximately half of these patients were followed by repeated CT scans $(n=31)$ and possibly later subjected to surgery if growth or morphological changes were observed. Within the average follow-up period of 11 months, two-thirds of all patients with negative or inconclusive ENB pathology underwent a diagnostic reintervention, with EBUS or pEBUS ( $n=13)$, CT-guided TTNA ( $\mathrm{n}=12$ ) or video-assisted thoracoscopic surgery (VATS) $(\mathrm{n}=15)$, which revealed another 20 malignant pathologies classified as false negatives (Figure 1).

Table 3 presents the performance of ENB in the first 81 consecutive procedures at our department. Twenty-one malignant lesions were found by ENB, however, after a mean follow-up of 11 months the total number of malignant cases amounted to 41 corresponding to a diagnostic yield and sensitivity of $51.2 \%$ (95\% CI: 35.1-67.1\%). The overall probability of correctly diagnosing a lesion, also named diagnostic accuracy, was $75.3 \%$ (95\% CI: 64.5-84.2\%).

Only one intrapulmonary haemorrhage occurred, which was conservatively managed. No clinical presentation of pneumothorax was observed; however subclinical cases cannot be ruled out because patients were not routinely subjected to a chest X-ray before discharge.

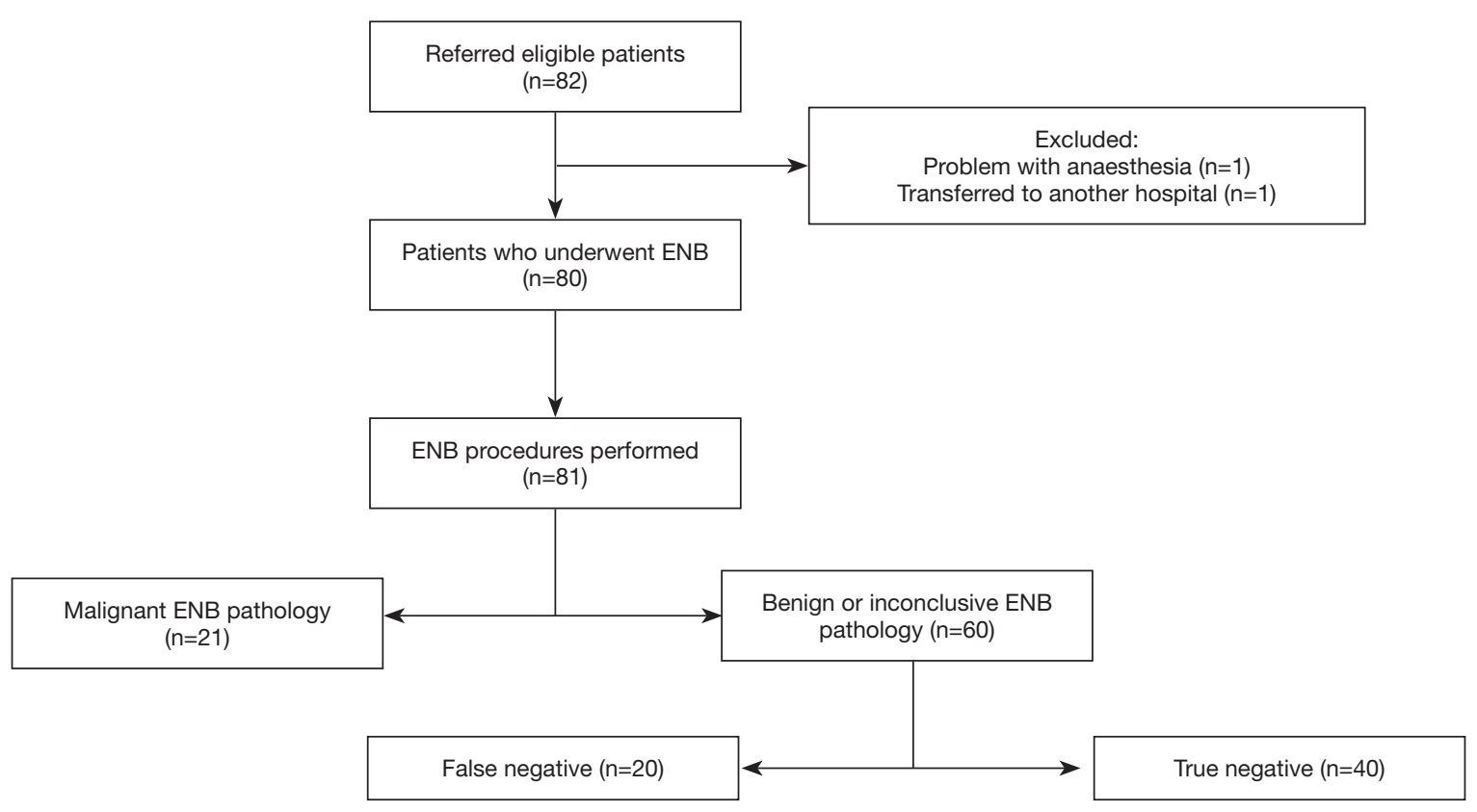

Figure 1 Flow diagram of all ENB procedures performed and corresponding pathology including the final results after a mean follow-up time of 11 months. ENB, electromagnetic navigation bronchoscopy. 
The learning curve of our initial series is illustrated in Figure 2 comparing the results based on diagnostic accuracy from the first consecutive $40 \mathrm{ENB}$ procedures with the last 41 procedures. An increase in the diagnostic accuracy from $67.5 \%$ to $82.9 \%$ and almost a half in the number of false negatives (13 vs. 7) was observed, however, the difference was not statistically significant (P value: 0.11 ).

Finally, logistic regression analysis revealed that previous cancer diagnosis does not predict a malignant ENB biopsy ( $\mathrm{P}$ value: 0.874$)$.

\section{Discussion}

This study evaluated the usefulness of adding ENB to the DLCDP as an adjunct diagnostic resource when traditional

Table 2 Pulmonary lesion characteristics $(n=81)$

\begin{tabular}{lc}
\hline Lesion location & $\mathrm{n}(\%)$ \\
\hline Right upper lobe & $35(43.2)$ \\
Right middle Lobe & $5(6.2)$ \\
Right lower Lobe & $10(12.3)$ \\
Left Upper lobe & $20(24.7)$ \\
Left lower lobe & $11(13.6)$ \\
Target size & \\
Diameter in cm, mean [SD] & $1.55[0.4]$ \\
Volume in cm ${ }^{3}$, mean [SD] & $1.64[1.8]$ \\
ENB Pathology & \\
Benign/inconclusive & $60(74.1)$ \\
Malignant & $21(25.9)$ \\
NSCLC & 17 \\
Other primary lung cancers & 1 \\
Metastasis & 3 \\
\hline
\end{tabular}

Location and pathology of the pulmonary lesions that underwent biopsy via ENB. NSCLC, non-small cell lung carcinoma. minimally invasive diagnostic methods had failed or were not amendable. Despite the highly selected and diagnostically challenging patient population, the results from our initial consecutive series of ENB procedures showed a diagnostic yield and accuracy of $51 \%$ and $75 \%$, respectively. Nevertheless, ENB was not capable of adequately excluding malignancy, as shown by a negative predictive value of $67 \%$ and negative likelihood ratio of 0.49 .

The reporting of the diagnostic yield and accuracy of ENB in the literature suffers from the lack of uniform definitions, and as outlined by several reviews and metaanalyses, it has indeed been reported with substantial variance ranging from $57 \%$ to $94 \%$ and $59 \%$ to $93 \%$, respectively $(6,8,9)$. Like any other diagnostic tool, the performance of ENB is dependent on many factors, in particular the settings and study population characteristics, which limits direct comparison of results across studies unless these factors are properly described and comparable (12).

Several factors related to the settings may impact outcomes. In the only randomised controlled trial concerning ENB, Eberhardt and colleagues investigated the benefit of combining different bronchoscopic modalities (13). Patients with solitary pulmonary nodules were randomised to one of three procedure groups: (I) ENB; (II) pEBUS; (III) ENB and pEBUS (in the latter pEBUS was only used for placement confirmation). The diagnostic yield was statistical significantly higher $(\mathrm{P}=0.02)$ when combining ENB and pEBUS, despite a significantly larger mean lesion size in the ENB group (28 vs. 25. vs. $24 \mathrm{~mm}$, ENB vs. EBUS vs. combined ENB-pEBUS, $\mathrm{P}=0.03)$. In this initial series a simple setup was preferred but meanwhile the combination of $\mathrm{ENB}$ and pEBUS has been implemented at our institution.

Furthermore, pooled outcome data has shown that the corresponding use of general anaesthesia and ROSE significantly increases the diagnostic yield and sensitivity for malignancy (6). As stated, ROSE was unfortunately not feasible at our institution and most likely it would have improved the ENB performance in the current series. However, we performed all ENB procedures using general

Table 3 Diagnostic performance of ENB

\begin{tabular}{|c|c|c|c|c|}
\hline ENB procedures (n) & $\begin{array}{l}\text { Diagnostic accuracy (\%) } \\
\qquad(95 \% \mathrm{Cl})\end{array}$ & $\begin{array}{c}\text { Diagnostic yield/ } \\
\text { sensitivity (\%) }(95 \% \mathrm{Cl})\end{array}$ & NPV (\%) $(95 \%$ Cl) & LR (95\% Cl) \\
\hline 81 & $75.3(64.5-84.2)$ & $51.2(35.1-67.1)$ & 66.7 (59.4-73.2) & $0.49(0.36-0.67)$ \\
\hline
\end{tabular}




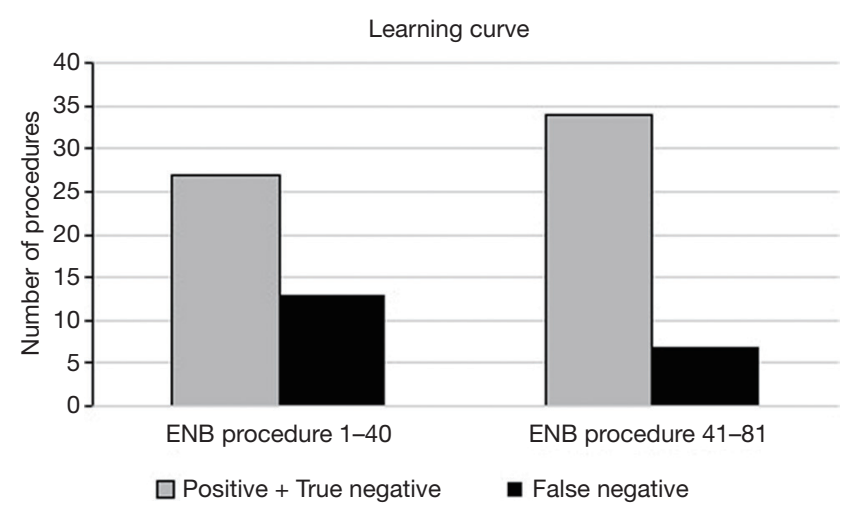

Figure 2 Bar chart displaying the learning curve based on diagnostic accuracy of our initial $81 \mathrm{ENB}$ procedure grouped into the first 40 consecutive procedures versus the last 41 procedures. ENB, electromagnetic navigation bronchoscopy.

anaesthesia, which we believe was of particularly great advantage in cases with difficult navigation and target alignment. Even so, a growing number of institutions are safely performing ENB under sedation with acceptable and non-inferior diagnostic results (14-16). We decided from the beginning not to use fluoroscopy based on the investigations by Eberhardt et al. (17) and as mentioned to make the setup as simple as possible. Interestingly, pooled results have later confirmed that studies, in which fluoroscopy were not applied, had significantly higher diagnostic yields (6).

The impact of the learning curve when introducing ENB must also not be underestimated. Lamprecht et al. (18) studied the diagnostic yield of 112 ENB procedures and observed a steep learning curve with an increase in diagnostic yield of $80 \%$ and $87.5 \%$ for, respectively, the first 30 and last 30 procedures. Likewise, the learning curve of our initial series showed a non-significant but substantial improvement in diagnostic accuracy and almost a half in the number of false negatives when comparing the results from the first $40 \mathrm{ENB}$ procedures with the last 41 procedures ( $\mathrm{P}$ value: 0.11$)$.

Since the majority of original articles concerning the diagnostic performance of ENB are relatively small retrospective single centre studies, the reported variability is obviously also largely driven by differences in patient selection, which arguably can be broken down to four key aspects: (I) lesion size; (II) lesion location and/or presence of bronchus sign; (III) ENB's priority in the diagnostic work-up pathway (first versus later when other diagnostic methods have failed); and (IV) prevalence of malignancy in the study population.

Previously reported lesion sizes (mean diameter) varies from $20 \mathrm{~mm}$ (19) to $39.8 \mathrm{~mm}$ (20), which makes the present mean diameter of $1.55 \mathrm{~cm}(\mathrm{SD}: 0.4 \mathrm{~cm})$ the lowest reported so far. This highlights our selection of the most difficult diagnostic cases and provides explanation to the correspondingly low diagnostic yield of $51 \%$. While some previous ENB investigations claim that the diagnostic yield was independent of lesion size $(13,18,21)$, other studies have proven a close relation between these two variables $(14,22)$.

Furthermore, lesions located in the lower lobes are associated with a lower yield $(13,14)$, however, the presence of bronchus sign on the CT scan significantly raised the diagnostic yield to $79 \%$ compared to $31 \%$ in cases where it was absent (22). In our series, 21/81 (25.9\%) of the lesions were located in the lower lobes and although we did not record the presence of bronchus sign (or CTratio and distance from the pleura), we do from experience concur with its positive impact on the diagnostic yield. Nevertheless, in this initial series of highly selected patients (generally not obvious surgical candidates) these factors had no impact on the MDT decision because a minimal invasive biopsy was desired. In this context, we highlight that we were not in a position where we could select the patients based on factors that would positively influence the diagnostic yield. But we were keen to know if the addition of ENB would be a true clinically applicable asset to our minimal invasive diagnostic algorithm and subsequently help us reduce potentially harmful or futile diagnostic surgical resections in frail patients. Consequently, we actually predisposed ENB to the most diagnostically challenging cases regardless of conventional recommendation of ENB suitability and obviously these data cannot be extrapolated to population screening or algorithms with ENB as the first choice of the diagnostic work up.

Additionally, the priority of ENB in the diagnostic work-up pathway may change the study population and cancer prevalence substantially, which ultimately impacts ENB performance. Though rarely explicitly stated in the articles, we argue that a non-negligible reason for the noted variability between studies could arise from differences in the priority of ENB in the diagnostic workup algorithm along with other diagnostic procedures. As displayed in the review by Gex et al. (6) the selection of patients for most studies were based on the presence of a peripheral pulmonary lesion beyond the field of the flexible bronchoscope, but generally no diagnostic method had previously been attempted. In contrast, we 
purposely prioritized ENB as a "last resource" before potential diagnostic wedge resection in the algorithm of diagnostic methods, which meant that $87.7 \%$ of the lesions subjected to ENB in our series had previously undergone an unsuccessful diagnostic biopsy. Moreover, two-thirds of the patients with negative or inconclusive ENB pathology underwent diagnostic re-intervention based on a new MDT decision. If the patient in spite of increased perioperative risks was found eligible for surgery, a VATS procedure was the natural next step of the diagnostic algorithm $(\mathrm{n}=15)$. However, if the patient was deemed a poor surgical candidate and the probability for malignancy was considered high, re-intervention with CT-guided TTNA $(n=12)$ or EBUD/pEBUS ( $n=13$ ) was elected to be the best possible minimal invasive option to get diagnostic reassurance despite previous failure or reservation regarding low yield. We acknowledge that the pathway of this patient subgroup is far from ideal, nevertheless, we believe it reflects daily clinical diagnostic challenges and dilemmas that many units can relate to.

In general, the studies with the highest diagnostic yields also have the highest prevalence of malignancy in the study population indicating a close relationship between these two variables $(13,18,23)$. The diagnostic yield/sensitivity of ENB in this study was low in accordance with a low prevalence of malignancy of $50.6 \%$. Likewise, Mahajan et al. applied the use of ENB in a high-risk patient population with a prevalence of malignancy of $57 \%$, which correspondingly revealed a low diagnostic yield of $49 \%$ (19).

The true prevalence of malignancy in the present patient population remains undetermined due to the average follow-up duration of 11 months. This must be noted as a limitation of the study because slow growing tumours might be missed on consecutive CT-scans and a longer follow up period would potentially impact the proportion of "true and false negatives". All included patients did, however, receive a PET-CT scan as part of their standard work-up, before any diagnostic method was applied, to investigate the possibility of distant metastases, recurrences or unknown primary tumour. Yet, a high proportion of previous cancer (71.6\%) of which NSCLC accounted for 31\% (18 out of 58; Table 1) was noted. But previous cancer history did not prove to be a predictor for malignant ENB pathology in our study. This result must, nevertheless, be interpreted with caution given the learning curve and the small number of procedures.

The high prevalence of previous cancer emphasizes that the main suspicion would frequently have been recurrence in a previously operated or radiated field rather than a new primary lung cancer. This might to some extent explain why traditional diagnostic methods were considered low yield or not amendable in $12.3 \%$ of the lesions included. Even so, this initial series revealed that at least $21 / 80$ patients $(26 \%)$ with true positive ENB pathology were indeed spared a potentially complicated surgical diagnostic procedure, which underlines the extended versatility and value that ENB can add to diagnostic work-up pathways.

To overcome the discussed differences in hospital settings and patient selection and provide better external validity of the performance of ENB, Folch and colleagues initiated the global prospective multicentre NAVIGATE study, which began enrolment in April 2015 (24). The recently published one-year results from the United States cohort comprising 1,215 ENB procedures, of which 976 (80.3\%) completed the 12-month follow-up, showed a diagnostic yield of $72.9 \%$ (range, 66.4-75.4\%) (25). The diagnostic yield in the NAVIGATE study was calculated as true positives plus true negatives divided by all attempted biopsies, which is equivalent to the definition of diagnostic accuracy used in our study revealing a comparable value of $75.3 \%$. However, the sensitivity and NPV of $68.8 \%$ and $56.3 \%$, respectively, found in the NAVIGATE study (25) were not exactly concordant with the present findings (Table 3), which might again be related to the relatively low prevalence of malignancy in our highly selected patient population.

Complications following ENB are rare as emphasized in a pooled analysis of 1,033 procedures showing an incidence of minor to moderate bleeding of $0.9 \%$ ( $95 \%$ CI: $0.4-1.6 \%$ ) and pneumothorax of 3.1\% (95\% CI: $2.1-$ $4.3 \%$ ). Moreover, only $1.6 \%$ (95\% CI: $1.0-2.6 \%$ ) required a chest tube insertion (6). We only encountered one moderate intrapulmonary bleeding, which was managed conservatively, and the patient recovered after a couple of days in the intensive care unit. No pneumothoraces were recorded, but the possibility of subclinical cases cannot be excluded since a chest $\mathrm{X}$-ray was not routinely performed before discharge. In contrast, pneumothorax after CTguided TTNA is quite common occurring in up to $43 \%$ of the patients, with $14 \%$ of patients requiring chest tube drainage (26). Furthermore, the risk of haemorrhage is approximately $5 \%(27,28)$. Thus, from a safety perspective this also makes ENB an attractive alternative to TTNA in frail patients with a high risk of pneumothorax.

\section{Conclusions}

We found ENB to be a safe and valuable asset to the 
DLCDP when used as an adjunct tool in diagnostically challenging patients in which other minimally invasive diagnostic procedures had failed or were impossible. The overall diagnostic performance was reflected by the learning curve and the highly selected cohort with a low prevalence of malignancy and the smallest target size yet reported. While further consideration needs to be given to which patient population ENB would benefit the most, we believe that the introduction of ENB as an additional diagnostic resource to the DLCDP has reduced the need of potentially high-risk and futile diagnostic surgical procedures at our institution.

\section{Acknowledgments}

The authors wish to thank the scrub nurses, operating theatre personal and all collaborating departments for their embracing commitment to the implementation of ENB at the Department of Cardiothoracic Surgery, Odense University Hospital. Also, a special thanks to research assistant Claire Zammit, School of Public Health and Preventive Medicine, Monash University for proof reading and revising the manuscript on English grammar and style.

Funding: None.

\section{Footnote}

Reporting Checklist: The authors have completed the STARD reporting checklist. Available at http://dx.doi.org/10.21037/ jtd-20-1236

Data Sharing Statement: Available at http://dx.doi. org/10.21037/jtd-20-1236

Peer Review File: Available at http://dx.doi.org/10.21037/jtd20-1236

Conflicts of Interest: All authors have completed the ICMJE uniform disclosure form (available at http://dx.doi. org/10.21037/jtd-20-1236). The authors have no conflicts of interest to declare.

Ethical statement: The authors are accountable for all aspects of the work in ensuring that questions related to the accuracy or integrity of any part of the work are appropriately investigated and resolved. This study was performed as a part of a quality assessment project; hence no informed consent of the participants was required. The study was conducted in accordance with the Declaration of Helsinki and approved by the local Ethics Committee at Odense University Hospital (case number: 18/12554).

Open Access Statement: This is an Open Access article distributed in accordance with the Creative Commons Attribution-NonCommercial-NoDerivs 4.0 International License (CC BY-NC-ND 4.0), which permits the noncommercial replication and distribution of the article with the strict proviso that no changes or edits are made and the original work is properly cited (including links to both the formal publication through the relevant DOI and the license). See: https://creativecommons.org/licenses/by-nc-nd/4.0/.

\section{References}

1. Sundhedsstyrelsen. Pakkeforløb for lungekræft - For fagfolk. Copenhagen: Danish Health Authority, 2018.

2. Garzon JC, Ng CS, Sihoe AD, et al. Video-assisted thoracic surgery pulmonary resection for lung cancer in patients with poor lung function. Ann Thorac Surg 2006;81:1996-2003.

3. McKenna RJ Jr, Mahtabifard A, Yap J, et al. Wedge resection and brachytherapy for lung cancer in patients with poor pulmonary function. Ann Thorac Surg 2008;85:S733-6.

4. Mehta AC, Hood KL, Schwarz Y, et al. The Evolutional History of Electromagnetic Navigation Bronchoscopy: State of the Art. Chest 2018;154:935-47.

5. Dhillon SS, Harris K. Bronchoscopy for the diagnosis of peripheral lung lesions. J Thorac Dis 2017;9:S1047-58.

6. Gex G, Pralong JA, Combescure C, et al. Diagnostic yield and safety of electromagnetic navigation bronchoscopy for lung nodules: a systematic review and meta-analysis. Respiration 2014;87:165-76.

7. Goud A, Dahagam C, Breen DP, et al. Role of electromagnetic navigational bronchoscopy in pulmonary nodule management. J Thorac Dis 2016;8:S501-8.

8. Muñoz-Largacha JA, Litle VR, Fernando HC. Navigation bronchoscopy for diagnosis and small nodule location. J Thorac Dis 2017;9:S98-103.

9. Zhang W, Chen S, Dong X, et al. Meta-analysis of the diagnostic yield and safety of electromagnetic navigation bronchoscopy for lung nodules. J Thorac Dis 2015;7:799-809.

10. Dale CR, Madtes DK, Fan VS, et al. Navigational bronchoscopy with biopsy versus computed tomography- 
guided biopsy for the diagnosis of a solitary pulmonary nodule: a cost-consequences analysis. J Bronchology Interv Pulmonol 2012;19:294-303.

11. Leong S, Ju H, Marshall H, et al. Electromagnetic navigation bronchoscopy: A descriptive analysis. J Thorac Dis 2012;4:173-85.

12. Whiting PF, Rutjes AW, Westwood ME, et al. A systematic review classifies sources of bias and variation in diagnostic test accuracy studies. J Clin Epidemiol 2013;66:1093-104.

13. Eberhardt R, Anantham D, Ernst A, et al. Multimodality bronchoscopic diagnosis of peripheral lung lesions: a randomized controlled trial. Am J Respir Crit Care Med 2007;176:36-41.

14. Bowling MR, Kohan MW, Walker P, et al. The effect of general anesthesia versus intravenous sedation on diagnostic yield and success in electromagnetic navigation bronchoscopy. J Bronchology Interv Pulmonol 2015;22:5-13.

15. Cheng SL, Chu CM. Electromagnetic navigation bronchoscopy under intravenous sedation-tips and tricks. J Thorac Dis 2018;10:S769-74.

16. Cheng SL, Chu CM. Electromagnetic navigation bronchoscopy: the initial experience in Hong Kong. J Thorac Dis 2019;11:1697-704.

17. Eberhardt R, Anantham D, Herth F, et al. Electromagnetic navigation diagnostic bronchoscopy in peripheral lung lesions. Chest 2007;131:1800-5.

18. Lamprecht B, Porsch P, Wegleitner B, et al. Electromagnetic navigation bronchoscopy (ENB): Increasing diagnostic yield. Respir Med 2012;106:710-5.

19. Mahajan AK, Patel S, Hogarth DK, et al. Electromagnetic navigational bronchoscopy: an effective and safe approach to diagnose peripheral lung lesions unreachable by conventional bronchoscopy in high-risk patients. J Bronchology Interv Pulmonol 2011;18:133-7.

Cite this article as: Stenger M, Zoorob S, Hussein AA, Eckardt J. Electromagnetic navigation bronchoscopy as an adjunct diagnostic tool in the Danish lung cancer diagnostic pathway: an initial retrospective single centre series. J Thorac Dis 2020;12(9):4762-4770. doi: 10.21037/jtd-20-1236
20. Becker HD, Herth F, Ernst A, et al. Bronchoscopic Biopsy of Peripheral Lung Lesions Under Electromagnetic Guidance: A Pilot Study. Bronchol 2005;12:9-13.

21. Gildea TR, Mazzone PJ, Karnak D, et al. Electromagnetic navigation diagnostic bronchoscopy: a prospective study. Am J Respir Crit Care Med 2006;174:982-9.

22. Seijo LM, de Torres JP, Lozano MD, et al. Diagnostic yield of electromagnetic navigation bronchoscopy is highly dependent on the presence of a Bronchus sign on CT imaging: results from a prospective study. Chest 2010;138:1316-21.

23. Eberhardt R, Morgan RK, Ernst A, et al. Comparison of suction catheter versus forceps biopsy for sampling of solitary pulmonary nodules guided by electromagnetic navigational bronchoscopy. Respiration 2010;79:54-60.

24. Folch EE, Bowling MR, Gildea TR, et al. Design of a prospective, multicenter, global, cohort study of electromagnetic navigation bronchoscopy. BMC Pulm Med 2016;16:60.

25. Folch EE, Pritchett MA, Nead MA, et al. Electromagnetic Navigation Bronchoscopy for Peripheral Pulmonary Lesions: One-Year Results of the Prospective, Multicenter NAVIGATE Study. J Thorac Oncol 2019;14:445-58.

26. Gould MK, Donington J, Lynch WR, et al. Evaluation of individuals with pulmonary nodules: when is it lung cancer? Diagnosis and management of lung cancer, 3rd ed: American College of Chest Physicians evidence-based clinical practice guidelines. Chest 2013;143:e93S-120S.

27. Boskovic T, Stanic J, Pena-Karan S, et al. Pneumothorax after transthoracic needle biopsy of lung lesions under CT guidance. J Thorac Dis 2014;6 Suppl 1:S99-107.

28. Yeow KM, Su IH, Pan KT, et al. Risk factors of pneumothorax and bleeding: multivariate analysis of 660 CT-guided coaxial cutting needle lung biopsies. Chest 2004;126:748-54. 